\title{
GM-CSF is not essential for experimental autoimmune encephalomyelitis but promotes brain-targeted disease
}

\author{
Emily R. Pierson and Joan M. Goverman \\ Department of Immunology, University of Washington, Seattle, Washington, USA.
}

Experimental autoimmune encephalomyelitis (EAE) has been used as an animal model of multiple sclerosis to identify pathogenic cytokines that could be therapeutic targets. Granulocytemacrophage colony-stimulating factor (CM-CSF) is the only cytokine reported to be essential for EAE. We investigated the role of CM-CSF in EAE in $\mathrm{C} 3 \mathrm{HeB} / \mathrm{Fe}$ ) mice that uniquely exhibit extensive brain and spinal cord inflammation. Unexpectedly, CM-CSF-deficient C3HeB/FeJ mice were fully susceptible to EAE because IL-17 activity compensated for the loss of GM-CSF during induction of spinal cord-targeted disease. In contrast, both GM-CSF and IL-17 were needed to fully overcome the inhibitory influence of IFN- $\gamma$ on the induction of inflammation in the brain. Both CM-CSF and IL-17 independently promoted neutrophil accumulation in the brain, which was essential for braintargeted disease. These results identify a CM-CSF/IL-17/IFN- $\gamma$ axis that regulates inflammation in the central nervous system and suggest that a combination of cytokine-neutralizing therapies may be needed to dampen central nervous system autoimmunity.

Conflict of interest: The authors have declared that no conflict of interest exists.

Submitted: December 16, 2016 Accepted: February 23, 2017

Published: April 6, 2017

\section{Reference information:} JCI Insight. 2017;2(7):e92362. https:// doi.org/10.1172/jci.insight.92362.

\section{Introduction}

Experimental autoimmune encephalomyelitis (EAE), a widely used animal model of multiple sclerosis (MS), is initiated by myelin-specific $\mathrm{CD}^{+} \mathrm{T}$ cells infiltrating the central nervous system (CNS). These $\mathrm{T}$ cells produce cytokines within the CNS that promote leukocyte recruitment, triggering an inflammatory cascade that ultimately results in tissue damage. Most mouse strains exhibit classic EAE in which parenchymal inflammation occurs predominantly in the spinal cord and is manifested as ascending flaccid paralysis. Previous studies showed that this spinal cord-predominant pattern of inflammation is a result of IFN- $\gamma$ production by infiltrating T cells, as IFN- $\gamma$ signaling promotes inflammation in the spinal cord but suppresses inflammation in the brain (1). We developed a unique model of $\mathrm{EAE}$ in $\mathrm{C} 3 \mathrm{HeB} / \mathrm{FeJ}$ mice that exhibits both parenchymal brain and spinal cord inflammation on a wild-type background. In addition to signs of classic EAE, these mice exhibit atypical clinical signs associated with brain inflammation that include ataxia, leaning, and rolling. Subsequent studies in this model showed that IL-17 signaling counteracted the suppressive effect of IFN- $\gamma$ in the brain, such that increasing the ratio of IL-17-secreting to IFN- $\gamma$-secreting T cells infiltrating the CNS promoted brain inflammation and atypical EAE $(2,3)$. Mechanistically, IL-17 promoted, but IFN- $\gamma$ inhibited, neutrophil recruitment to the brain, and neutrophil infiltration was essential for the development of atypical, but not classic, EAE (3). Neutrophils appear to play an important role in promoting leukocyte migration out of perivascular cuffs (3); however, once leukocytes gain access to the brain parenchyma, other cell types such as monocytes may also contribute to tissue injury (4).

While IFN- $\gamma$ and IL-17 clearly play important roles in determining where lesions localize within the CNS, genetic deficiency of each cytokine showed that they were both dispensable for classic EAE induction $(1,5)$. In contrast, multiple published studies concluded that granulocyte-macrophage colony-stimulating factor (GM-CSF) is an essential T cell cytokine in EAE. Several strains of mice deficient in GM$\mathrm{CSF}$, including NOD/Lt, B10.PL, and C57BL/6 are extremely resistant to EAE (6-9), and treatment with recombinant GM-CSF exacerbated disease (6). GM-CSF functions as a hematopoietic growth factor and proinflammatory cytokine, promoting activation and tissue accumulation of monocytes, macrophages, and granulocytes $(10,11)$. Although a variety of cell types can produce GM-CSF, including macrophages and endothelial cells (10), adoptive transfer experiments showed that GM-CSF produced by myelin-specific $\mathrm{T}$ cells, and not host cells, was essential to induce EAE in B10.PL and C57BL/6 mice (7-9). A pathogenic 
role for GM-CSF has also been suggested in MS, as higher frequencies of GM-CSF-producing T cells have been found in the cerebrospinal fluid and blood of MS patients compared with controls (12-14).

The requirement for GM-CSF in EAE was demonstrated only in models of classic EAE; therefore, the contribution of GM-CSF to brain inflammation was not determined. We took advantage of the fact that wild-type $\mathrm{C} 3 \mathrm{HeB} / \mathrm{FeJ}$ mice develop both brain and spinal cord inflammation to investigate whether GM-CSF was essential for atypical EAE. We backcrossed the GM-CSF knockout mutation (Csf2-1-) onto the $\mathrm{C} 3 \mathrm{HeB} / \mathrm{FeJ}$ background and induced EAE by adoptive transfer to investigate the effect of GM-CSF deficiency in myelin-specific $\mathrm{T}$ cells. To our surprise, recipients of $C S f 2^{-/-}$donor T cells developed normal classic EAE. We found that deficiency in either GM-CSF or the IL-17 receptor (IL-17R) did not affect the incidence or severity of classic EAE; however, classic EAE was impaired when signaling from both cytokines was lacking. The redundant activities of GM-CSF and IL-17 in promoting spinal cord-targeted disease were independent of neutrophil recruitment. In contrast, T cell production of GM-CSF independently promoted atypical EAE, and this activity was dependent on neutrophil recruitment.

\section{Results}

We investigated the contribution of GM-CSF to EAE in wild-type $\mathrm{C} 3 \mathrm{HeB} / \mathrm{FeJ}$ mice by adoptively transferring $\mathrm{T}$ cells isolated from wild-type and $\mathrm{Cs}_{\mathrm{f}} 2^{-/-}$mice immunized with recombinant myelin oligodendrocyte glycoprotein (MOG) into wild-type recipient mice. T cells isolated from donor mice were cultured with IL-23 prior to transfer to maximize their potential to produce IL-17 and induce atypical EAE, and equivalent numbers of antigen-specific (blasting) donor $\mathrm{CD}^{+} \mathrm{T}$ cells from wild-type and $\mathrm{Cs}_{\mathrm{f}} 2^{-{ }_{-}}$mice were transferred into recipient mice. We focused on this adoptive transfer approach because $\mathrm{T}$ cell priming, especially of Th17 cells, is less efficient in $C s f 2^{-/-}$compared with wild-type mice $(6,15,16)$, which confounds interpretation of the specific contribution of GM-CSF in models of active EAE induction. Surprisingly, mice that received $\mathrm{Csf}^{-1-}$ donor T cells developed EAE with comparable incidence and severity as mice that received wild-type $\mathrm{T}$ cells (Figure 1 and Table 1 ). While recipients of $C s f 2^{-1-} \mathrm{T}$ cells had a significantly later day of onset and slightly slower progression, there was no significant difference in the maximum EAE scores between the 2 groups (Figure 1 and Table 1 ). We also transferred both wild-type and $C_{s f 2^{-1-}} \mathrm{T}$ cells into $C s f 2^{-1-}$ recipients. The $C s f 2^{-1-}$ recipients of each type of T cell developed nearly identical EAE to the wild-type recipients of the same donor cells (Figure 1), confirming previous reports that host expression of GM-CSF is not required for EAE induction (7). Thus, while differences in EAE onset and progression were observed when donor T cells lacked GM-CSF, these data indicate that GM-CSF production by either donor $\mathrm{T}$ cells or host cells is not required for induction of severe $\mathrm{EAE}$ in $\mathrm{C} 3 \mathrm{HeB} / \mathrm{FeJ}$ mice.

Although the overall incidence and severity of EAE was comparable when disease was induced by either $C s f 2^{--}$or wild-type T cells, the manifestation of EAE differed when donor T cells lacked GM-CSF. Among mice with EAE, both the incidence and severity of atypical EAE signs were significantly decreased among recipients of $\mathrm{Csf}^{-1-} \mathrm{T}$ cells compared with wild-type T cells (Table 1 ). In contrast, the incidence and severity of classic EAE signs was nearly identical among recipients of either $C s f 2^{-/}$or wild-type $\mathrm{T}$ cells, indicating a greater requirement for GM-CSF in promoting brain inflammation compared with spinal cord inflammation in $\mathrm{C} 3 \mathrm{HeB} / \mathrm{FeJ}$ mice.

We previously showed that the incidence of atypical but not classic EAE in $\mathrm{C} 3 \mathrm{HeB} / \mathrm{FeJ}$ mice was reduced when wild-type T cells were transferred into IL-17R-deficient $\left(I l 17 \mathrm{ra}^{-/}\right)$compared with wild-type mice, indicating that IL-17 plays a key role in promoting brain but not spinal cord inflammation (3). Importantly, it has been shown that $\mathrm{CD}^{+} \mathrm{T}$ cells primed in $\mathrm{Cs} f 2^{-/-}$mice produce lower levels of IL-17 upon restimulation compared with $\mathrm{T}$ cells primed in wild-type mice $(15,16)$. Consistent with these reports, we found that the frequency of $\mathrm{IL}-17^{+}$MOG-specific $\mathrm{CD}^{+} \mathrm{T}$ cells was reduced among $\mathrm{T}$ cells primed in $\mathrm{Cs}_{S} 2^{-1-}$ mice compared with $\mathrm{T}$ cells primed in wild-type mice, while the frequency of IFN- $\gamma^{+} \mathrm{T}$ cells was the same for both types of mice (Figure 2A). This suggested that reduced levels of IL-17 rather than lack of GM-CSF production by $C s f 2^{-1-} \mathrm{T}$ cells could account for the decrease in atypical EAE signs seen in wild-type recipient mice. To determine if GM-CSF has a role in promoting atypical EAE independent of IL-17 signaling, we transferred MOG-specific T cells from wild-type and $C s f 2^{-1-}$ mice into $I l 17 \mathrm{ra}^{-/-}$and wild-type recipients. We reasoned that if GM-CSF does not play an independent role in promoting atypical EAE, then there should be no further decrease in the incidence of atypical EAE in the $I l 17 \mathrm{ra}^{-/-}$recipient mice following transfer of $C s f 2^{--}$compared with wild-type T cells. As expected, the incidence of atypical EAE was reduced when wild-type donor T cells were transferred into $I 117 \mathrm{ra}^{-/-}$compared with wild-type recipients, while both 
Table 1. Incidence and severity of classic and atypical EAE induced by adoptive transfer of WT and Csf2 ${ }^{-/-}$T cells into WT, Csf2 ${ }^{-/-}$, and III7ra-/- recipient mice

\begin{tabular}{|c|c|c|c|c|c|c|}
\hline Adoptive Transfer & Overall Incidence & Mean day of onset ${ }^{A}$ & Percentage classic ${ }^{\mathrm{B}}$ & $\begin{array}{c}\text { Mean max classic } \\
\text { EAE score }^{A}\end{array}$ & Percentage atypicalB & $\begin{array}{c}\text { Mean max atypical } \\
\text { EAE score }\end{array}$ \\
\hline $\mathrm{WT} \rightarrow \mathrm{WT}$ & $100(60 / 60)$ & $6.2 \pm 0.1$ & 100 & $4.9 \pm 0.1$ & 94 & $4.5 \pm 0.1$ \\
\hline $\mathrm{Csf2^{-/- } \rightarrow \mathrm { WT }}$ & $98(55 / 56)$ & $7.2 \pm 0.1^{\complement}$ & 100 & $4.6 \pm 0.1$ & $58^{c}$ & $3.2 \pm 0.1^{\complement}$ \\
\hline $\mathrm{WT} \rightarrow\left[\mathrm{s} f 2^{-/-}\right.$ & $100(11 / 11)$ & $6.3 \pm 0.2$ & 100 & $5.1 \pm 0.1$ & 91 & $3.9 \pm 0.4$ \\
\hline $\mathrm{Csf2} 2^{-/-} \rightarrow \mathrm{Csf2} 2^{-/-}$ & $87(13 / 15)$ & $7.5 \pm 0.2$ & 100 & $4.5 \pm 0.2$ & $54^{D}$ & $3.1 \pm 0.3$ \\
\hline $\mathrm{WT} \rightarrow I / 17 \mathrm{ra}^{-/-}$ & $90(36 / 40)$ & $6.2 \pm 0.1$ & 100 & $4.7 \pm 0.2$ & $72^{\mathrm{E}}$ & $3.6 \pm 0.2^{F}$ \\
\hline$\left[\mathrm{sf2} 2^{-/-} \rightarrow I / 17 \mathrm{ra}^{-/-}\right.$ & $58(22 / 38)^{\complement}$ & $8.1 \pm 0.3^{H}$ & 100 & $1.9 \pm 0.2^{\prime}$ & $0^{\prime}$ & $0^{\prime}$ \\
\hline
\end{tabular}

${ }^{A}$ Data shown (mean \pm SEM) are from mice with experimental autoimmune encephalomyelitis (EAE), pooled from 13 experiments. ${ }^{\text {B } P e r c e n t a g e ~ c l a s s i c ~}$ or atypical is the percentage of mice with EAE exhibiting classic or atypical signs. ${ }^{C} P<0.0001$ compared with WT $\rightarrow$ WT. ${ }^{\mathrm{D}} P=0.001$ compared with $\mathrm{WT} \rightarrow \mathrm{WT}$. ${ }^{\mathrm{E}} P=0.003$ compared with WT $\rightarrow$ WT. ${ }^{\mathrm{F}} P<0.01$ compared with WT $\rightarrow$ WT. ${ }^{\mathrm{C}} P=0.002$ compared with $\mathrm{WT} \rightarrow / 117 \mathrm{ra}^{-1-}$ and $P<0.0001$ compared

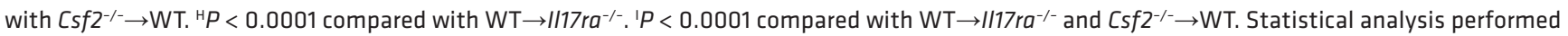
using a $\chi^{2}$ test (incidence and percentage classic or atypical), 1-way ANOVA with Tukey's post test (day of onset), or Kruskal-Wallis with Dunn's post test (classic or atypical EAE score).

Figure 1. GM-CSF is not required for $\mathrm{EAE}$ in $\mathrm{C} 3 \mathrm{HeB} / \mathrm{FeJ}$ mice. Mean clinical scores for mice that developed EAE following transfer of IL-23-conditioned T cells from MOG-immunized WT or $C s 2^{-/-}$donors into WT or $C s 2^{-1-}$ recipients. Data are pooled from 3 experiments in which WT T cells were transferred into

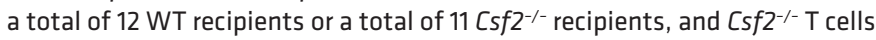
were transferred into a total of 12 WT recipients or a total of $13 \mathrm{Cs}^{-/-}$recipients. Mean clinical scores ( \pm SEM) were determined using a single numerical scoring system that includes both atypical and classic signs as described in Methods. EAE, experimental autoimmune encephalomyelitis; GM-CSF, granulocyte-macrophage colony-stimulating factor; MOC, myelin oligodendrocyte glycoprotein.

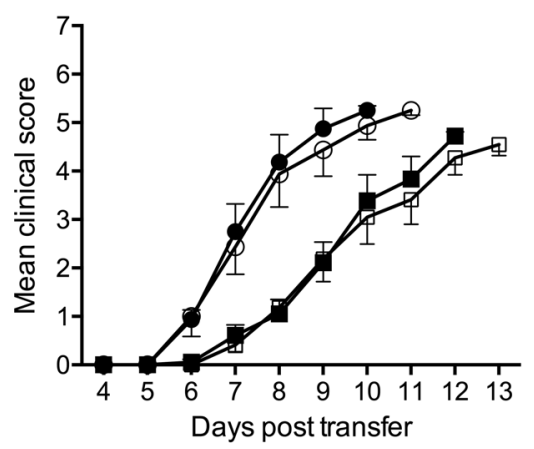


A
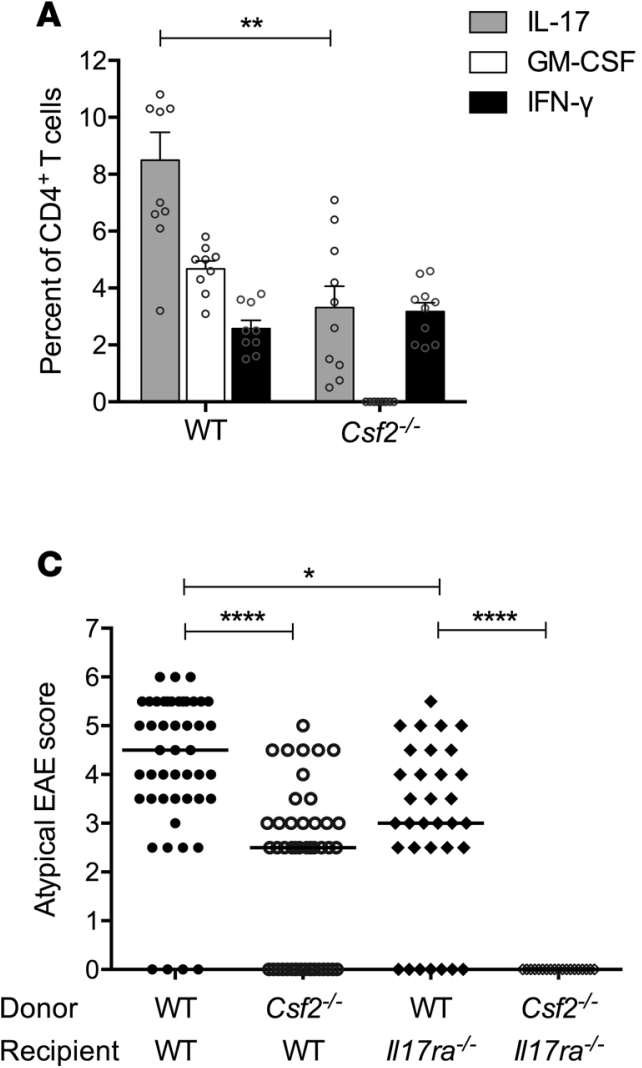

B
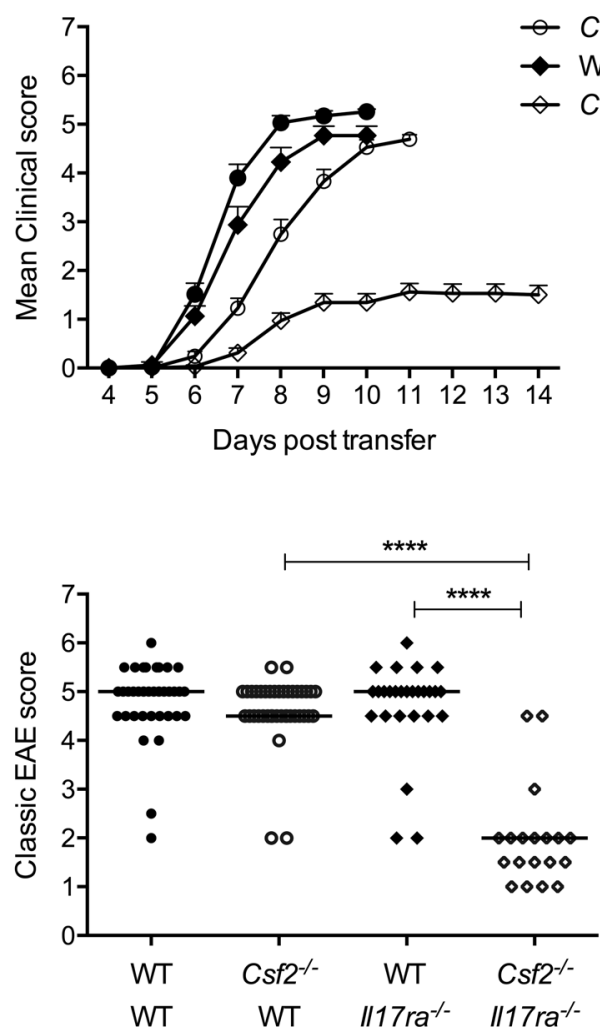

Figure 2. GM-CSF and IL-17 cooperate to induce both brain- and spinal cord-targeted disease. (A) Percentage of WT or Csf2- donor CD4 ${ }^{+}$T cells producing

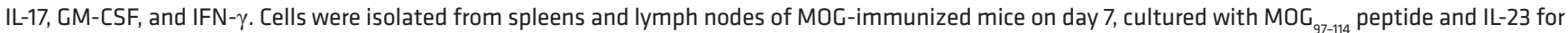
3 days, and stimulated in vitro with $\mathrm{MOG}_{97-114}$ for 4 hours prior to intracellular staining and flow cytometric analysis. Data (mean \pm SEM) were pooled from 9 experiments. (B) Mean clinical scores for mice that developed EAE induced by adoptive transfer of WT and Csf2 $2^{-/-}$donor CD4+ ${ }^{+}$cells into WT or I/17ra ${ }^{-/-}$recipients. Mean clinical scores were determined using the combined scoring system as in Figure 1. Data (mean \pm SEM) were pooled from 9 experiments with 35 $\mathrm{WT} \rightarrow \mathrm{WT}, 35 \mathrm{Csf2}^{-/-} \rightarrow \mathrm{WT}, 24 \mathrm{WT} \rightarrow I 117 \mathrm{ra}^{-1-}$, and $20 \mathrm{Csf2}^{-1-} \rightarrow 1117 \mathrm{ra}^{-1-}$. (C) The maximum scores for atypical signs (left) and classic signs (right) are shown for individual mice from the experiments graphed in $\mathbf{B} .{ }^{*} P<0.05$, ${ }^{* *} P<0.01$, ${ }^{* * *} P<0.0001$ by Student's $t$ test (A) or Kruskal-Wallis with Dunn's post test (C). EAE, experimental autoimmune encephalomyelitis; CM-CSF, granulocyte-macrophage colony-stimulating factor; MOC, myelin oligodendrocyte glycoprotein.

recipient mice (Figure 3A). Notably, transfer of donor $C_{s f 2^{--}}$T cells with the highest IL-17/IFN- $\gamma$ ratio induced atypical EAE in all recipient mice, indicating that GM-CSF production by donor T cells is not

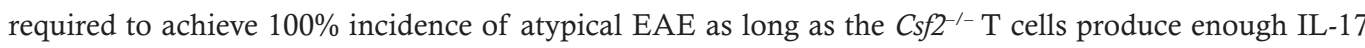
relative to IFN- $\gamma$. We then assessed whether the GM-CSF/IFN- $\gamma$ ratio of wild-type donor T cells transferred into IL-17R-deficient recipients also positively correlated with the incidence of atypical EAE. We compiled data from 12 EAE experiments in which the GM-CSF/IFN- $\gamma$ ratio of wild-type donor T cells ranged from 0.5 to 2.0 prior to transfer into $I l 17 \mathrm{ra}^{-/-}$recipients. Again, a significant positive correlation was observed between the GM-CSF/IFN- $\gamma$ ratios of the wild-type donor T cells and the incidence of atypical EAE in the $1117 \mathrm{ra}^{-/-}$recipients (Figure 3B), indicating that IL-17 signaling is not required to initiate atypical EAE if donor T cells produce sufficient GM-CSF relative to IFN- $\gamma$. However, the severity of atypical EAE in mice lacking either GM-CSF or IL-17 signaling was lower than the severity seen in wild-type controls (Table 1), even in experiments where the incidence of atypical EAE was 100\% (data not shown). Thus, in wild-type mice, it is likely that the activities of IL-17 and GM-CSF synergize to achieve severe inflammation in the brain.

Previous studies have shown that neutrophil recruitment to the brain is critical for the parenchymal tissue damage that causes the clinical signs of atypical $\operatorname{EAE}(3,17,18)$. Therefore, we investigated whether the decrease in atypical EAE induced by $C_{S} f^{--}$compared with wild-type $\mathrm{T}$ cells was associated with a decrease in neutrophil accumulation in the brain. Fewer neutrophils accumulated in the brain of Ill $\mathrm{ra}^{-1}$ compared with wild-type recipients of wild-type $\mathrm{T}$ cells at peak EAE (Figure 4A), consistent with our previous results (3). A similar decrease in neutrophil numbers was also observed in wild-type recipients 
A

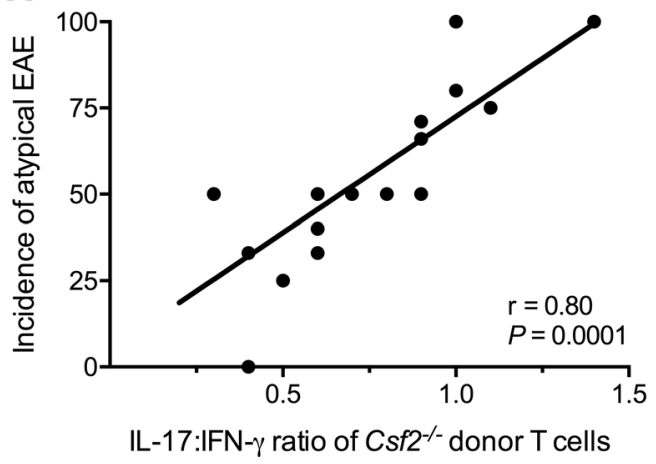

B

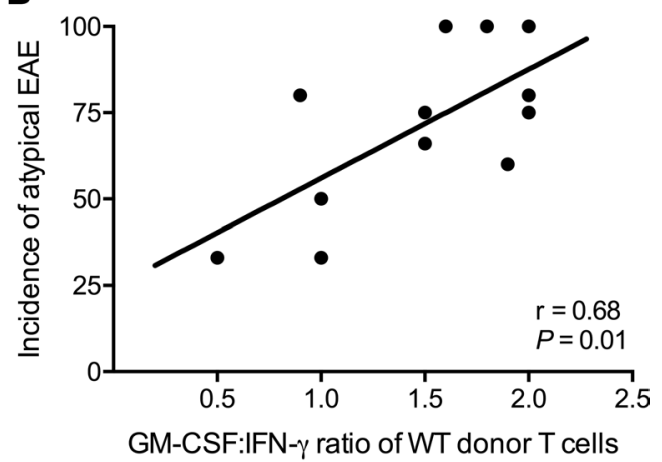

Figure 3. Increasing the expression of either IL-17 or GM-CSF alone can overcome the inhibitory influence of IFN- $\gamma$ on atypical EAE. (A) The incidence of atypical EAE observed in 16 different experiments in which $C s 2^{-1-}$ T cells were transferred into WT recipients is plotted against the IL-17/IFN- $\gamma$ ratio of the donor cells prior to transfer for each experiment. (B) The incidence of atypical EAE observed in 12 different experiments in which WT T cells were transferred into $/ 177 \mathrm{ra}^{-/-}$recipients is plotted against the GM-CSF/IFN- $\gamma$ ratio of the donor T cells prior to transfer for each experiment. (A and B) Intracellular cytokine staining of the $C D 4^{+} T$ cells in each experiment was performed on the day of transfer, as described in Methods. Incidence of atypical EAE is shown as a percentage of sick mice. Pearson correlation analysis was used to determine $r$ correlation coefficients and $P$ values. EAE, experimental autoimmune encephalomyelitis; CM-CSF, granulocyte-macrophage colony-stimulating factor.

of $C s f 2^{-1-} \mathrm{T}$ cells compared with recipients of wild-type $\mathrm{T}$ cells. Importantly, combining the deficiencies in donor T cell-derived GM-CSF and recipient IL-17 signaling resulted in an additional significant decrease in neutrophil numbers in both the brain and spinal cord (Figure 4A), as well as a complete loss of atypical EAE (Table 1). The number of neutrophils observed in the absence of both GM-CSF and IL-17R was equivalent to that seen in naive animals (data not shown). The decrease in neutrophil numbers when wildtype versus $\mathrm{CS}_{\mathrm{S} 2^{--}-}$donor T cells were transferred into $\mathrm{IlI}_{\mathrm{Tr}}{ }^{-/-}$recipients indicates that GM-CSF independently promotes neutrophil accumulation.

Interestingly, while the numbers of neutrophils that accumulated in the brains of mice lacking only T cell-derived GM-CSF or lacking only IL-17 signaling were lower than the low end of the range seen in wild-type mice (Figure 4A), more than half of these mice still exhibited some atypical clinical signs. This suggested that low numbers of neutrophils were sufficient for development of mild atypical EAE. To confirm that these small numbers of neutrophils were required to induce this mild atypical EAE, we depleted neutrophils from recipient mice by administering the neutrophil-specific anti-Ly6G antibody. Consistent with our prior observations (3), atypical EAE induced by wild-type T cells in wild-type recipients is strongly attenuated when neutrophils are depleted (Figure 4B). Importantly, when atypical EAE was driven either by IL-17 (donor T cells lacked GM-CSF), or by GM-CSF (recipients of wild-type T cells lacked IL-17R), neutrophil depletion again strongly attenuated atypical EAE signs (Figure 4B). These data suggest that GM-CSF and IL-17 act synergistically to promote brain-targeted disease by enhancing the accumulation of neutrophils in the brain. In the spinal cord, even though the neutrophil numbers were decreased in the absence of either GM-CSF or IL-17R, classic EAE was not dependent on neutrophil recruitment (Figure 4B), in agreement with our earlier studies (3).

\section{Discussion}

The data presented here contradict the current paradigm that GM-CSF is an essential cytokine in the pathogenesis of EAE. In contrast to the requirement for GM-CSF seen in other classic EAE models (6-9), we found that GM-CSF and IL-17 play redundant roles with each other in promoting classic EAE in $\mathrm{C} 3 \mathrm{HeB} /$ FeJ mice, such that only the combined deficiency of both cytokines had any effect on disease incidence and severity. It is possible that deficiency in GM-CSF but not IL-17 confers resistance to classic EAE in other mouse models because these strains may generate fewer IL- $17^{+}$myelin-specific $\mathrm{T}$ cells compared with $\mathrm{C} 3 \mathrm{HeB} / \mathrm{FeJ}$ mice. Our previous studies in $\mathrm{C} 3 \mathrm{HeB} / \mathrm{FeJ}\left(\mathrm{H}-2^{\mathrm{k}}\right) \times \mathrm{C} 3 \mathrm{H} . \mathrm{SW}\left(\mathrm{H}-2^{\mathrm{b}}\right) \mathrm{F} 1$ mice, which present both the $\mathrm{H}-2^{\mathrm{k}}-$ restricted $\mathrm{MOG}_{97-114}$ epitope targeted in $\mathrm{C} 3 \mathrm{HeB} / \mathrm{FeJ}$ mice and the $\mathrm{H}-2^{\mathrm{b}}$-restricted $\mathrm{MOG}_{35-55}$ epitope also targeted in $\mathrm{C} 57 \mathrm{BL} / 6$ mice, support this hypothesis. We found that $\mathrm{F} 1$ mice generated a greater number of IL-17+ $\mathrm{T}$ cells responding to the $\mathrm{MOG}_{97-114}$ epitope than to the $\mathrm{MOG}_{35-55}$ epitope, although both epitopes elicited similar numbers of IFN $-\gamma^{+} \mathrm{T}$ cells (ref. 2 and unpublished data). Therefore, strains that 
A

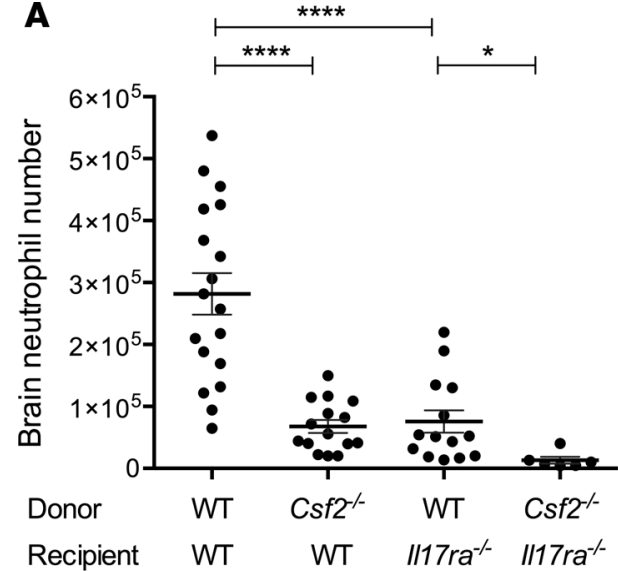

B

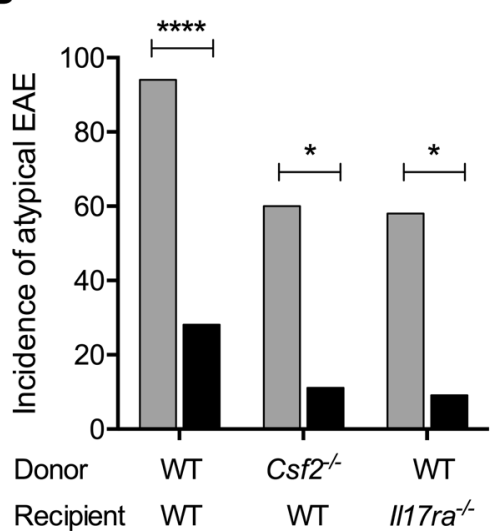

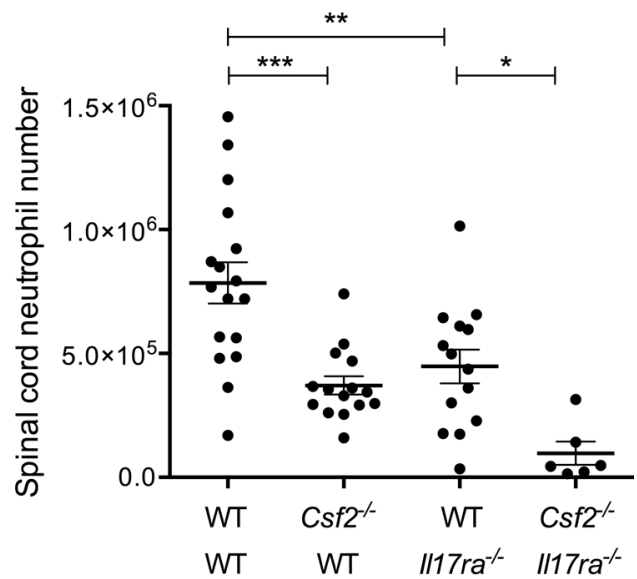

Isotype

$\alpha-L y 6 G$

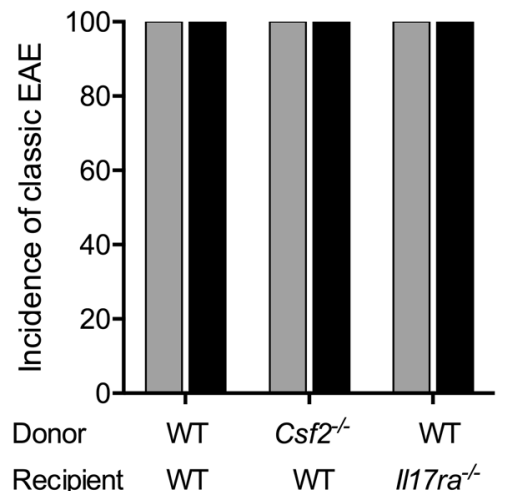

Figure 4. GM-CSF and IL-17 independently promote neutrophil accumulation that is required to induce atypical EAE. (A) Numbers of neutrophils (CD11 $\mathrm{b}^{+} \mathrm{CD} 45^{+} \mathrm{Ly} 6 \mathrm{C}^{+}$) were determined by flow cytometric analyses of cells isolated from brains (left) and spinal cords (right) of WT and $1 / 17 \mathrm{ra}^{-1-}$ recipients of either WT or $\mathrm{Csf2}^{-1-} \mathrm{CD} 4^{+} \mathrm{T}$ cells at peak EAE. Data were pooled from 6 experiments. Error bars represent SEM. (B) Incidence of atypical (left) or classic (right) EAE signs in WT or II17ra-/- recipient mice that were treated with either isotype control or anti-Ly6G antibody after transfer of WT or $\mathrm{Csf2} 2^{-1-} \mathrm{CD} 4^{+} \mathrm{T}$ cells. Incidence of classic or atypical signs is shown as a percentage of sick mice. Data are compiled from 4

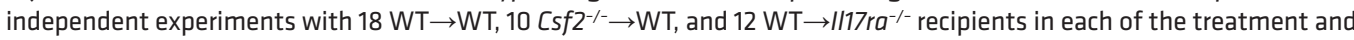
control groups. ${ }^{*} P<0.05,{ }^{* *} P<0.01,{ }^{* * *} P<0.001,{ }^{* * *} P<0.0001$ by 1 -way ANOVA with Tukey's post test (A) or $\chi^{2}$ test (B). EAE, experimental autoimmune encephalomyelitis; GM-CSF, granulocyte-macrophage colony-stimulating factor.

generate fewer IL-17+ myelin-specific T cells would have increased reliance on GM-CSF+ $\mathrm{T}$ cells to induce spinal cord inflammation.

The only significant effect on classic EAE seen in the absence of donor T cell-derived GM-CSF was a 1-day delay in disease onset compared with wild-type mice. We speculate that the delayed onset may reflect the need for a threshold level of total proinflammatory cytokine production (GM-CSF, IL-17, IFN- $\gamma$ ) within the localized region of the perivascular space to trigger the inflammatory cascade that ultimately leads to parenchymal spinal cord infiltration. When the infiltrating donor T cells produce only IL-17 and IFN- $\gamma$ but not GM-CSF, more time may be needed to exceed this threshold requirement for proinflammatory cytokines. However, once the threshold is reached, inflammation is initiated and normal classic disease severity is attained.

Although we found that both IL-17 and GM-CSF independently promoted neutrophil recruitment to the spinal cord in $\mathrm{C} 3 \mathrm{HeB} / \mathrm{FeJ}$ mice (Figure 4A), neutrophil depletion did not affect the incidence or severity of classic EAE in wild-type or IL-17R-deficient recipients that received either wild-type or $\mathrm{Csf2}_{\mathrm{f}}{ }^{-1}$ donor $\mathrm{T}$ cells. Thus, both IL-17 and GM-CSF promote spinal cord-targeted disease via a neutrophil-independent mechanism, as we previously observed for IFN- $\gamma$ (3). In C57BL/6 mice, expression of the GM-CSF receptor only on $\mathrm{CCR} 2^{+}$cells and not $\mathrm{LysM}^{+}$cells (including neutrophils) was required to induce classic $\mathrm{EAE}$, 
suggesting that monocyte activation by GM-CSF is critical to initiate spinal cord inflammation (19). CCR2 ${ }^{+}$ monocytes clearly play an important role in promoting classic EAE, but they were not completely essential in every model $(17,20)$, and CCR2 antagonists have not been effective in MS clinical trials $(11,21)$. We observed a moderate decrease in the number of monocytes in the brain and spinal cord of $\mathrm{C} 3 \mathrm{HeB} / \mathrm{FeJ}$ mice with EAE when donor T cells lacked GM-CSF (Supplemental Figure 2), but there was no corresponding decrease in incidence or severity of classic EAE in the absence of GM-CSF alone (Table 1). However, in the absence of both donor T cell GM-CSF and recipient IL-17R, the numbers of both neutrophils and monocytes were sharply decreased in the spinal cord (Figure 4A and Supplemental Figure 2) and classic EAE was attenuated, suggesting that infiltration of at least one of these cell types is needed for spinal cord disease.

In contrast to the normal classic EAE induced by $C_{s f} 2^{-1-} \mathrm{T}$ cells in wild-type recipients, development of atypical EAE in the same mice was impaired. We found that GM-CSF plays a similar role as IL-17 in countering the suppressive effects of IFN- $\gamma$ in the brain. Accordingly, the incidence of atypical EAE in IL-17R-deficient recipient mice positively correlated with the GM-CSF/IFN- $\gamma$ ratio of wild-type donor T cells. Notably, a 100\% incidence of atypical EAE occurred in IL-17R-deficient mice at higher GM-CSF/ IFN- $\gamma$ ratios (Figure 3B). We were surprised by the overall higher incidence of atypical EAE in $I l 17 \mathrm{ra}^{-1}$ recipients of wild-type $\mathrm{T}$ cells in these experiments compared with the incidence that we observed in similar experiments in our previous study (3), although we still observed a significant decrease in atypical EAE in $I l 17 \mathrm{ra}^{-/-}$compared with wild-type recipients (Table 1). Because our earlier experiments were conducted in a different animal facility, we compared historical data for cytokine production by the MOG-specific T cells generated in the previous facility to results obtained from mice immunized in our current facility. We observed an increase in GM-CSF but similar IFN- $\gamma$ production by MOG-specific T cells from mice housed in our current facility compared with our previous facility. This change resulted in a significant increase in the GM-CSF/IFN- $\gamma$ ratios for donor T cells used in the experiments reported here (Supplemental Figure 3 ), which correlates with the increase in atypical EAE incidence in $1117 \mathrm{ra}^{-1-}$ mice. Thus, in our previous animal colony where less GM-CSF was produced relative to IFN- $\gamma$, IL-17 was likely more important for promoting development of atypical EAE. Together, these findings indicate that the balance of both IL-17 and GM-CSF relative to IFN- $\gamma$ is critical in regulating atypical EAE.

IL-17, GM-CSF, and IFN- $\gamma$ appear to influence the development of atypical EAE primarily by regulating accumulation of neutrophils in the brain. We previously demonstrated that IL-17 promotes and IFN- $\gamma$ inhibits neutrophil recruitment to the brain (3), and we find here that GM-CSF also promotes neutrophil accumulation in the brain and spinal cord independent of IL-17 signaling. While GM-CSF could potentially influence EAE by activating and recruiting different types of myeloid cells, neutrophils were required for the GM-CSF-driven atypical EAE that developed in IL-17R-deficient mice (Figure 4B), supporting the notion that GM-CSF, like IL-17, promotes atypical EAE through neutrophil recruitment. GM-CSF could influence neutrophil recruitment by directly or indirectly upregulating ELR ${ }^{+}$CXC chemokines (22) or neutrophil $N$-formyl-L-methionyl-L-leucyl-phenylalanine (fMLP) receptors (23), or by acting as a direct chemoattractant for neutrophils (24). GM-CSF can also delay neutrophil apoptosis, induce neutrophil priming and activation, and induce neutrophil production of cytokines such as IL-8 and IL-1 $\beta$ (25-27). The incidence and severity of atypical EAE clearly correlated with the number of neutrophils in the brain, as wild-type mice exhibited severe atypical EAE and the greatest accumulation of neutrophils, mice lacking either IL-17R or GM-CSF had reduced neutrophil accumulation in the brain and only half of the mice developed mild atypical EAE, while mice lacking both GM-CSF and IL-17R had no neutrophil recruitment and exhibited no atypical EAE. Thus, production of both cytokines was necessary to fully overcome IFN- $\gamma$-mediated suppression of neutrophil recruitment and induce the severe atypical EAE seen in wildtype $\mathrm{C} 3 \mathrm{HeB} / \mathrm{FeJ}$ mice. Our finding that only a small number of neutrophils was sufficient to initiate mild atypical EAE is consistent with the fact that neutrophils are not a predominant feature on brain sections from patients with MS.

In summary, our results suggest that there is no single $\mathrm{T}$ cell cytokine that is essential for EAE induction. While multiple cytokines may contribute to the pathogenesis of CNS inflammation, we propose that the overall balance of GM-CSF, IL-17, and IFN- $\gamma$ plays a critical role in determining the induction and manifestation of disease. Both IL-17 and GM-CSF have been implicated as pathogenic cytokines in MS (12-14, 28, 29), and clinical trials targeting individual cytokines with anti-IL-17A and anti-GM-CSF monoclonal antibodies are ongoing. We suggest that a combination of therapies might be necessary, as the importance of either cytokine may vary among individual patients. 


\section{Methods}

Mice. $\mathrm{C} 3 \mathrm{HeB} / \mathrm{FeJ}$ mice were originally purchased from The Jackson Laboratory and were bred and maintained in a specific pathogen-free facility at the South Lake Union Campus of the University of Washington. GM-CSF knockout ( $C S f 2^{--}$) mice (30) were obtained from Jay Heinicke (University of Washington, Seattle) and were backcrossed in our facility onto the $\mathrm{C} 3 \mathrm{HeB} / \mathrm{FeJ}$ background for at least 14 generations. Ill $7 \mathrm{ra}^{-1-}$ mice generated by Amgen were obtained from Taconic and were backcrossed onto the $\mathrm{C} 3 \mathrm{HeB} / \mathrm{FeJ}$ background for 20 generations.

Protein and peptides. Rat recombinant MOG (rMOG) protein (amino acids 1-125) was produced in Escherichia coli and purified as previously described (31). MOG $_{97-114}$ peptide (TCFFRDHSYQEEAAVELK) was synthesized by GenScript.

EAE induction. EAE was induced by adoptive transfer using mice between 6 and 12 weeks of age. Donor mice were subcutaneously immunized with $100 \mu \mathrm{g}$ of rMOG emulsified in complete Freund's adjuvant (CFA, Sigma-Aldrich) and cells were isolated from the spleen and draining lymph nodes 7 days later. Cells were cultured at $1 \times 10^{7}$ cells/ml for 3 days with $\mathrm{MOG}_{97-114}$ peptide $(10 \mu \mathrm{M})$ and $10 \mathrm{ng} / \mathrm{ml} \mathrm{rIL}-23$ (R\&D Systems). Viable cells were isolated from a Lympholyte gradient (Cedarlane) and the frequency of $\mathrm{CD}^{+} \mathrm{T}$ cell blasts $\left(\mathrm{CD}^{+} \mathrm{FSC}^{\mathrm{hi}} / \mathrm{SSC}^{\text {hi }}\right.$ cells) as well as their cytokine expression was analyzed by flow cytometry. $\mathrm{CD}^{+} \mathrm{T}$ cell blasts $\left(3 \times 10^{5}\right)$ were intraperitoneally (i.p.) injected into sublethally irradiated $(250$ $\mathrm{rad}$ ) recipient mice. EAE clinical signs were scored as follows: grade 1, paralyzed tail and/or hindlimb clasping; grade 2, head tilt and/or hindlimb weakness; grade 3, one paralyzed leg and/or mild body leaning; grade 4, two paralyzed legs and/or moderate body leaning; grade 5, forelimb weakness and/or severe body leaning; grade 6, hunched posture and/or breathing difficulty and/or body rolling; grade 7, moribund. Atypical EAE was identified by the presence of 1 or more of the following sign(s): head tilt, body leaning, or body rolling. An overall daily score was assigned based on the maximal severity of either classic or atypical signs seen in each mouse. The separate maximum severity of classic or atypical signs was also calculated for each mouse, as reported in Figure 2C and Table 1.

Isolation of CNS mononuclear cells. Mononuclear cells were isolated from the CNS after cardiac perfusion with PBS as previously described (32). Briefly, brains and spinal cords were dissociated through sterile stainless steel mesh and centrifuged at $4^{\circ} \mathrm{C}$ for 10 minutes at 2,000 g. Cell pellets were resuspended in $30 \%$ Percoll, overlaid onto 70\% Percoll, and centrifuged without brake at room temperature for 20 minutes at $1,500 \mathrm{~g}$. Cells were collected from the $30 \%-70 \%$ Percoll interface.

Flow cytometry. Cells were first incubated with a fixable cell viability dye (Molecular Probes) for 20 minutes at $4^{\circ} \mathrm{C}$. After washing, cells were incubated with Fc block (clone $2.4 \mathrm{G} 2$; eBioscience) for 15 minutes at $4^{\circ} \mathrm{C}$ in $5 \%$ normal mouse serum and stained with fluorochrome-conjugated monoclonal antibodies for 30 minutes at $4^{\circ} \mathrm{C}$. Intracellular cytokine staining was performed after stimulation of cells for 4 hours with $\mathrm{MOG}_{97-114}$ peptide $(1 \mu \mathrm{M})$, according to the manufacturer's directions (BD Biosciences). Antibodies specific for CD11b (M1/70), GM-CSF (MP1-22E9), and the isotype control for GM-CSF (rat IgG2a, $\kappa$ ) were from eBioscience; antibodies specific for CD45 (30-F11), CD4 (RM4-5), Ly6G (1A8), Ly6C (AL-21), IL-17A (TC11-18H10) and its isotype control (rat IgG1, $\kappa)$, and IFN- $\gamma$ (XMG1.2) and its isotype control (rat IgG1, $\kappa$ ) were from BD Biosciences. Cells were analyzed using a FACSCanto cytometer (BD Biosciences) and FlowJo software (Tree Star).

Neutrophil depletion. The neutrophil-depleting anti-Ly6G antibody (clone 1A8) and isotype control antibody (clone 2A3) were purchased from Bio X Cell. Beginning on the day of T cell transfer, $200 \mu \mathrm{g}$ of either anti-Ly6G or isotype control antibody was administered by i.p. injection every other day until time of sacrifice as previously described (3).

Statistics. Statistical analyses were performed with Prism version 6.0 (GraphPad Software). Clinical scores were compared using the Kruskal-Wallis test with Dunn's multiple comparisons post test. Day of onset and cell numbers were compared using the 1-way ANOVA with Tukey's multiple comparisons post test. Cytokine frequencies were compared using the unpaired 2-tailed Student's $t$ test. Frequencies (incidence) of clinical signs were compared using the chi-squared test. The Pearson $r$ correlation coefficient was calculated where indicated. A $P$ value less than 0.05 was considered significant.

Study approval. This study was approved by the IACUC at the University of Washington in Seattle.

\section{Author contributions}

EP planned and performed experiments, and wrote the manuscript. JG supervised the study and wrote the manuscript. 


\section{Acknowledgments}

We thank Neal Mausolf for technical assistance and Catriona Wagner and Pamela Roqué for careful reading of the manuscript. This work was supported by a grant to J.M. Goverman from the National Institute of Allergy and Infectious Diseases (R37 AI107494-01).

Address correspondence to: Joan Goverman, Department of Immunology, University of Washington, Box 358059, 750 Republican Street, Seattle, Washington 98109-8509, USA. Phone: 206.685.7604; E-mail: goverman@uw.edu.

1. Miller NM, Wang J, Tan Y, Dittel BN. Anti-inflammatory mechanisms of IFN- $\gamma$ studied in experimental autoimmune encephalomyelitis reveal neutrophils as a potential target in multiple sclerosis. Front Neurosci. 2015;9:287.

2. Stromnes IM, Cerretti LM, Liggitt D, Harris RA, Goverman JM. Differential regulation of central nervous system autoimmunity by $\mathrm{T}(\mathrm{H}) 1$ and $\mathrm{T}(\mathrm{H}) 17$ cells. Nat Med. 2008;14(3):337-342.

3. Simmons SB, Liggitt D, Goverman JM. Cytokine-regulated neutrophil recruitment is required for brain but not spinal cord inflammation during experimental autoimmune encephalomyelitis. J Immunol. 2014;193(2):555-563.

4. Mangalam AK, et al. Absence of IFN- $\gamma$ increases brain pathology in experimental autoimmune encephalomyelitis-susceptible DRB1*0301.DQ8 HLA transgenic mice through secretion of proinflammatory cytokine IL-17 and induction of pathogenic monocytes/microglia into the central nervous system. J Immunol. 2014;193(10):4859-4870.

5. Haak S, et al. IL-17A and IL-17F do not contribute vitally to autoimmune neuro-inflammation in mice. J Clin Invest. 2009;119(1):61-69.

6. McQualter JL, et al. Granulocyte macrophage colony-stimulating factor: a new putative therapeutic target in multiple sclerosis. J Exp Med. 2001;194(7):873-882.

7. Ponomarev ED, Shriver LP, Maresz K, Pedras-Vasconcelos J, Verthelyi D, Dittel BN. GM-CSF production by autoreactive T cells is required for the activation of microglial cells and the onset of experimental autoimmune encephalomyelitis. J Immunol. 2007;178(1):39-48.

8. Codarri L, et al. ROR $\gamma \mathrm{t}$ drives production of the cytokine GM-CSF in helper T cells, which is essential for the effector phase of autoimmune neuroinflammation. Nat Immunol. 2011;12(6):560-567.

9. El-Behi M, et al. The encephalitogenicity of T(H)17 cells is dependent on IL-1- and IL-23-induced production of the cytokine GM-CSF. Nat Immunol. 2011;12(6):568-575.

10. Shi Y, et al. Granulocyte-macrophage colony-stimulating factor (GM-CSF) and T-cell responses: what we do and don't know. Cell Res. 2006;16(2):126-133.

11. Croxford AL, Spath S, Becher B. GM-CSF in neuroinflammation: Licensing myeloid cells for tissue damage. Trends Immunol. 2015;36(10):651-662.

12. Hartmann FJ, et al. Multiple sclerosis-associated IL2RA polymorphism controls GM-CSF production in human TH cells. Nat Commun. 2014;5:5056.

13. Noster R, et al. IL-17 and GM-CSF expression are antagonistically regulated by human Thelper cells. Sci Transl Med. 2014;6(241):241ra80.

14. Rasouli J, et al. Expression of GM-CSF in T cells is increased in multiple sclerosis and suppressed by IFN- $\beta$ therapy. J Immunol. 2015;194(11):5085-5093.

15. Sonderegger I, Iezzi G, Maier R, Schmitz N, Kurrer M, Kopf M. GM-CSF mediates autoimmunity by enhancing IL-6-dependent Th17 cell development and survival. J Exp Med. 2008;205(10):2281-2294

16. Ko HJ, et al. GM-CSF-responsive monocyte-derived dendritic cells are pivotal in Th17 pathogenesis. J Immunol. 2014;192(5):2202-2209.

17. Stoolman JS, Duncker PC, Huber AK, Segal BM. Site-specific chemokine expression regulates central nervous system inflammation and determines clinical phenotype in autoimmune encephalomyelitis. J Immunol. 2014;193(2):564-570

18. Liu Y, Holdbrooks AT, Meares GP, Buckley JA, Benveniste EN, Qin H. Preferential recruitment of neutrophils into the cerebellum and brainstem contributes to the atypical experimental autoimmune encephalomyelitis phenotype. J Immunol. 2015;195(3):841-852.

19. Croxford AL, et al. The cytokine GM-CSF drives the inflammatory signature of CCR2+ monocytes and licenses autoimmunity Immunity. 2015;43(3):502-514.

20. Gaupp S, Pitt D, Kuziel WA, Cannella B, Raine CS. Experimental autoimmune encephalomyelitis (EAE) in CCR2(-/-) mice: susceptibility in multiple strains. Am J Pathol. 2003;162(1):139-150.

21. Braddock M, Murray C. 10th anniversary Inflammation and Immune Diseases Drug Discovery and Development Summit. 20-21 March 2006, New Brunswick, USA. Expert Opin Investig Drugs. 2006;15(6):721-727.

22. McDermott AJ, Frank CR, Falkowski NR, McDonald RA, Young VB, Huffnagle GB. Role of GM-CSF in the inflammatory cytokine network that regulates neutrophil influx into the colonic mucosa during Clostridium difficile infection in mice. Gut Microbes. 2014;5(4):476-484.

23. Weisbart RH, Golde DW, Gasson JC. Biosynthetic human GM-CSF modulates the number and affinity of neutrophil f-MetLeu-Phe receptors. J Immunol. 1986;137(11):3584-3587.

24. Khajah M, Millen B, Cara DC, Waterhouse C, McCafferty DM. Granulocyte-macrophage colony-stimulating factor (GMCSF): a chemoattractive agent for murine leukocytes in vivo. J Leukoc Biol. 2011;89(6):945-953.

25. Coxon A, Tang T, Mayadas TN. Cytokine-activated endothelial cells delay neutrophil apoptosis in vitro and in vivo. A role for granulocyte/macrophage colony-stimulating factor. J Exp Med. 1999;190(7):923-934.

26. Wright HL, Moots RJ, Bucknall RC, Edwards SW. Neutrophil function in inflammation and inflammatory diseases. Rheumatology 
(Oxford). 2010;49(9):1618-1631.

27. Yao Y, Matsushima H, Ohtola JA, Geng S, Lu R, Takashima A. Neutrophil priming occurs in a sequential manner and can be visualized in living animals by monitoring IL-1 $\beta$ promoter activation. J Immunol. 2015;194(3):1211-1224.

28. Tzartos JS, et al. Interleukin-17 production in central nervous system-infiltrating $\mathrm{T}$ cells and glial cells is associated with active disease in multiple sclerosis. Am J Pathol. 2008;172(1):146-155.

29. Brucklacher-Waldert V, Stuerner K, Kolster M, Wolthausen J, Tolosa E. Phenotypical and functional characterization of T helper 17 cells in multiple sclerosis. Brain. 2009;132(Pt 12):3329-3341.

30. Dranoff G, Mulligan RC. Activities of granulocyte-macrophage colony-stimulating factor revealed by gene transfer and gene knockout studies. Stem Cells. 1994;12 Suppl 1:173-82; discussion 182.

31. Abdul-Majid KB, et al. Screening of several H-2 congenic mouse strains identified H-2(q) mice as highly susceptible to MOGinduced EAE with minimal adjuvant requirement. J Neuroimmunol. 2000;111(1-2):23-33.

32. Brabb T, von Dassow P, Ordonez N, Schnabel B, Duke B, Goverman J. In situ tolerance within the central nervous system as a mechanism for preventing autoimmunity. J Exp Med. 2000;192(6):871-880. 